\title{
A geologically-sound representation for evolutionary multi-objective
}

\section{subsurface identification}

\author{
Vijay Pratap Singh \\ Institut Français du Pétrole \\ Exploration-Production Business Unit \\ 1 et 4, avenue de Bois-Préau, 92852 \\ Rueil-Malmaison, Cedex France \\ Email: V-Pratap.SINGH@ifp.fr
}

\author{
Marc Schoenauer \\ LRI - Bat. 490 \\ Université Paris Sud \\ 91405 Orsay Cedex, FRANCE \\ Phone: +33(0) 169156626 \\ Email marc@lri.fr
}

\author{
Michel Léger \\ Institut Français du Pétrole \\ Exploration-Production Business Unit \\ 1 et 4, avenue de Bois-Préau, 92852 \\ Rueil-Malmaison Cedex, France \\ michel.leger@ifp.fr
}

\begin{abstract}
A new representation for evolutionary subsurface identification from surface or well geological data is proposed. The idea is to represent the subsurface as the combination of, first, a geologically initial set of horizontal layers and, second, fault parameters like shape and displacements. Based on volume and bed-length preservation, a morphogenesis process then gives the structure at present time. A first implementation of this representation is tested on an artificial geological inverse problem in foothills region: the fault locations and dips are considered as two different objectives, and the $\varepsilon$-MOEA multiobjective evolutionary algorithm is applied. The first results show the efficiency of the chosen representation.
\end{abstract}

\section{Introduction}

Since petroleum is gradually getting rare, oil companies prospect in new areas like foothills. Because of the rugged topography, the presence of many folds and faults, it is difficult to determine the geologic structures, either by seismic imaging or geological methods.

The problem is hence to identify the geological characteristics of the subsurface from sparse data. The available information can come from either surface geologic surveying or well logging, and consists in layer identification in nature and orientation. The reconstruction of the subsurface thus amounts to an identification problem.

The traditional approach for geological modelling repeatedly evaluates geological models with respect to balancing principles, namely, rock volume and bedlength preservation. These geometrical hypotheses, which are only approximations to reality, mean that geologic structures behave locally like a folded paperback book, with layers slipping on each other like pages during folding. This geological modelling, which may be backward (Moretti et al., 1989) or forward (Endignoux et al., 1989), is very cumbersome and human time consuming.

Geophysical modelling, on the other hand, has taken advantage from the progresses of numerical modelling, and several works address the problem using classical gradient-based identification methods. Because the problem either has to be oversimplified, or is ill-posed, several works used Evolutionary Algorithms (EAs), that are known to be well suited for solving complex, mathematically ill-posed identification problems (Schoenauer and Sebag, 2002). However, a critical question is the choice of a representation (and of the associated variation operators (crossover and mutation)). In the case of subsurface identification, state-of-the-art works either assumed some expert knowledge about the geometry of the subsurface structure (e.g. horizontal layers, or more complex geologically-driven topology (Boschetti et al., 1996)), or used global automatic models that lack geological soundness (Mansanné et al., 2002) (e.g. the subsurface structures are not balanced).

In this paper, we propose an original indirect representation for subsurface identification. The morphogenesis process (from the encoded representation to the present subsurface structure) only generates balanced geometries. Moreover, it can be used to solve either or both the geological and the geophysical identification problems by EAs automatically, and without the need for any expert 
knowledge. The idea is to represent a subsurface structure by the combination of, first, an initial configuration (series of flat homogeneous layers of different geological nature), second, some initial faults in that simple structure, and third, a description of successive geological deformations along the ages. A kinematic model, that relates fold geometry to fault shape and displacement, is used to construct crosssections of the geological structures. These crosssections are automatically balanced by virtue of the above-mentioned balancing principles (volume and bed-length preservation) upon which the kinematic model is based. The resulting complex subsurface structure is then used to compute the identification criteria: comparison with surface or subsurface geological data, geophysical simulation of some seismic experiment, etc.

However, as already mentioned, the different types of available information may be conflicting. Therefore the problem is better reformulated into a multiobjective optimization problem: there is no unique optimal solution but a set known as the Pareto set of optimal solutions, also termed non-dominated non-inferior, admissible, or efficient solutions. In this paper, multiobjective evolutionary algorithms (MOEAs), more precisely, the Epsilon-MOEA [Deb-epsilon], have been used to tackle this multiobjective problem.

The paper is organized as follows: first the geological framework is presented in section 2, together with the kinematic model and the complete geological inverse problem is described. Section 3 introduces the original indirect representation and the variation operators, as well as the different fitness criteria. Section 4 gives the first experimental results on two artificial geological problems, involving respectively 3 and 7 faults, and discusses the limitation of such purely geological experiments. Finally, section 5 sketches the directions of research that the proposed approach opens.

\section{The geological problem}

\subsection{Geology}

Complexity and ambiguity of mountain fronts are what makes them the most challenging fronts for a geologist or a geophysicist. Now the thrust regions (mountainous regions) are the next human quest for petroleum exploration. These fold-and-thrust belts are the manifestation of collision of tectonic plates. Most fold-and-thrust belts consist of foreland-progressing and hinterland-dipping thrusts (Boyer and Elliott, 1982) that may connect layer-parallel detachments (décollement horizons). The distribution of mesoscopic deformation features in fault-related folds has important consequences for hydrocarbon migration, trapping and production. Structural, environmental and stratigraphic factors control the spatial distribution and intensity of the deformation features (e.g. Fischer and Jackson, 1999) of mountains. Under a given rheological stratigraphy (Woodward and Rutherford, 1989) and prevalent environmental conditions (Stewart and Alvarez, 1991; Jamison, 1992; Lemiszki et al., 1994). It is fault-fold kinematics which controls the distribution and intensity of deformation that develop in a rock sequence (Fischer et al., 1992; Storti and Salvini, 1996).

\subsection{Kinematic Models}

For the representation of mountainous structure mainly three types of kinematics models appear in the literature. 1) kink model (Suppe 1983, 1990), 2) trishear model (Erslev, 1991; Hardy and Ford, 1997; Allmendinger, 1998 ) and 3) force fold model (Johnson and Johnson, 2000). Amongst the above mentioned three types the kink model is considered best suited for thin skinned tectonic regions. Since we are interested in such regions, hence kink model is being used.

A two-dimensional geometric model of fault-bend folding for a thin-skinned tectonic was first formulated by Suppe (1983) based on the conservation of layer thickness and bed length. He showed its applicability in the simulation of the Pine Mountain thrust sheet in the 
southern Appalachians, and to the fold and thrust belt of Western Taiwan. The use of kink-band style folding with non-deforming footwalls (characteristic of such geometric models) has been criticized by Ramsay (1991) as being unrealistic. Another fault-bend folding model was proposed by Contreras and Suiter (1990), however it was also based on the conservation of area. Since their introduction, these geometric models have received a lot of attention (Medwedeff, 1989; Jordan et al., 1993) and have been used extensively to predict thrust fault geometries at depths based on observed fold geometries. They have also been used in a forward modeling sense to predict hanging wall geometries above thrust faults (Mitra, 1990; Contreras and Suiter, 1990, 1997; Zoetemeijer, 1993; Hardy S., 1995).

\subsection{The Contreras Model}

The kinematic model used in the current simulation was introduced by Contreras and Suiter (1990). This model can be applied to regions being deformed by shortening, extension and also for the duplex systems (Contreras and Suiter, 1997). This is a two-dimensional model and is based on a coordinate transformation from less deformed state to a more deformed state. The medium is subdivided into domains of constant dip and homogeneous displacement vector fields that are delimited by the planes bisecting the fault inflections. The displacement occurs by translation. The displacement trajectory is of constant length for all the displaced particles throughout the medium and is parallel to the underlying active fault segment. This model also considers fault parallel simple shearing. A complete derivation Contreras fault bend folding (FBF) was provided by Contreras and Suiter (1990). The heterogeneity of the displacement vector field across axial planes introduces longitudinal and angular shear strains. Transformations from one state to another state do not cause a change in area and also the deformation is isochoric, a characteristic typical of deformation by simple shear (Truesdell and Toupin, 1960).

\section{The Evolutionary Algorithm}

\subsection{The Representation}

As mentioned in the introduction, the main difficulty when tackling a geological or geophysical identification of subsurface structure problem by EAs is to define a representation: structured representations are either unrealistic in complex regions like foothills (e.g. a fixed number of horizontal layers) or require some very specific expert knowledge about the geometry of the subsurface structure (Boschetti et al., 1996). Unstructured representations, on the other hand, don't require any input from the expert - but they lack of the minimal geological common-sense and hence can lead to quasi-perfect numerical fit that are absurd from a geological point of view (Mansanné et al., 2002). Note that some alternative possibilities have been proposed, that define a global model with numerous local parameters (e.g. using spline nodes uniformly spread on the considered domain), but such representations have the same lack of geological soundness, while additionally resulting in a huge optimization problem for the sake of precision.

Moreover, it seems very difficult indeed to try to constrain the solution proposed by unstructured representations with some geological rules (e.g. some simple rules could be that the underground velocity should increase with the depth - but interesting regions are precisely regions where such rule is violated!).

The representation proposed in this work deals with the above-mentioned problem by relying on a kinematic model of subsurface deformation from an initial simple state (horizontal layers, that can be assumed to be universally true in all regions at some point in the past) and subsequent deformations based on one of the kinematic models discussed in section 2.2 that ensure the consistency of the structure at any time, including the final state of the model - the target subsurface structure.

In EAs terminology, the genotype space, in which the algorithm will actually search, and where the variation operators are defined, is here the space of 
initial configurations plus initial faults plus series of deformations (see figure 1 for the detailed description of the complex data-type). The phenotype space, or behavioral space, where the fitness of each genotype is computed, is the space of subsurface structures, obtained from the genotypes by applying a transformation, called the morphogenesis process, or also the numerical Contreras model described in section 2 . The tectonic modelling presented in section 2 is sound because we will obtain only balanced subsurface structures by this morphogenesis process.

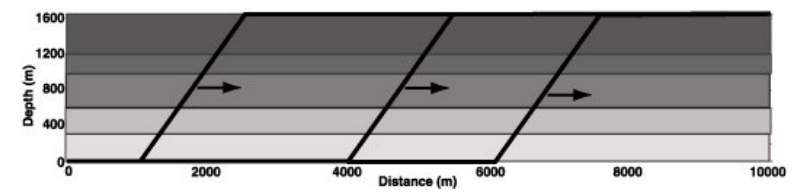

Figure 1 A chromosome: Unknown parameters are the number of initial layers and, for each layer, its thickness; the number of faults and, for each fault, its origin, and number of segment, and, for each segment, its length and dip; and the displacements (for each fault, one horizontal displacement).

\subsection{Initialization and Variation Operators}

First note that the representation proposed in this work is possibly a variable length representation (variable dimension parameter space). However, in the first experiments presented in section 3, we have used a fixed length representation: the number of faults and the number of segments per fault will be fixed (from the target model). We shall hence here only describe the operators of this fixed-length restriction.

\section{Initialization}

Here a complex structure of real values is being used, therefore the parameters are initialized uniformly on a given interval, with some geometrical constraints:

- Displacement of a fault can not be more than the difference of distance between the first segments of two successive faults.

- The length of first segment increases in the successive faults.

\section{Crossover Operator}

Because the representation is at the moment fixed length, the Simulated Binary Crossover (SBX) (Deb and Agrawal 95) recommended with the epsilonMOEA algorithm has been used. During the crossover, the geometry constraints listed above are respected.

A quick parameter study (12 independent runs for each value of $\eta$ ) has been performed (on the 3-faults problem described in section 4). All final populations are merged and non-domination sorting is performed (result can be seen in Figure 2). It is found that high value of $\eta$ is most suitable for this simulation, and thereon the distribution index $\eta$ has been set to 15 . Note that the recommended values of $\varepsilon_{1}=0.05$ and $\varepsilon_{2}=$ 0.05 were found robust enough and used in all experiments presented in section 4 .

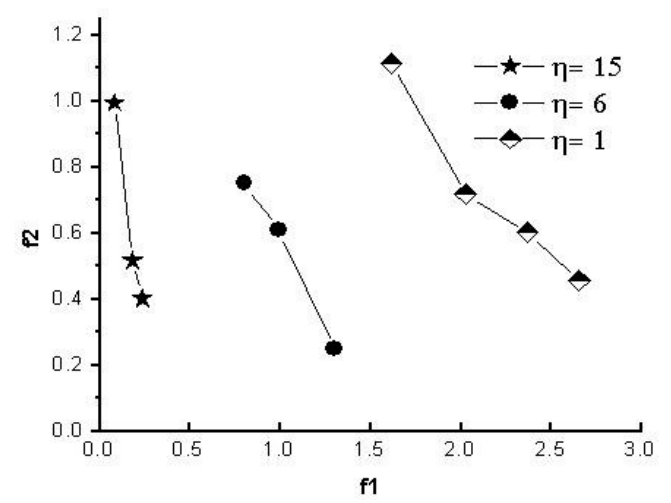

Figure 2 Influence of the distribution index $\eta$ of $S B X$ crossover on the results (results of 12 runs).

However, because the SBX crossover can hardly be applied on variable-length genotypes, some more variable-length specific crossover operators are used in the variable-length case (not described here for space reasons).

\section{Mutation Operator}

The SBX crossover is known to be selfadaptive in the sense that the spread of the possible offspring solutions depends on the distance between the parents, and decreases as the population converges (Beyer and Deb 2001). Hence it is generally used without any mutation (the use of mutation together with SBX in the context of the problems presented in 
section 4 did not seem to bring any benefit indeed, and was abandoned).

However, because the variable-length case requires additional use of mutation, self-adaptive Gaussian mutation (à la ES) is used on all real-valued parameters, while specific variable-length mutation operators are used to modify the numbers of faults and segments in each fault.

\subsection{The $\varepsilon$-Multiobjective Evolutionary Algorithm}

As mentioned in the introduction, various sources of data will be used for identification. Hence, the identification problems will be turned into MultiObjective Problems (MOPs). Examples of such problems can be seen in section 4.2 for the geological modeling problem, or in [Mansanné] for purely geophysical identification using both the semblance and the least-squares error on seismic signals. But the ultimate goal of the present work is to simultaneously use all available data, both geological and geophysical, leading to even more objectives.

During the past decade, various multiobjective evolutionary algorithms (MOEAs) have been proposed. A comprehensive review are given by Coello et al., (1999); Veldhuizen (2000); Deb (2002). Although these MOEAs differ from each other in both exploitation and exploration, they share the common purpose: searching for a near-optimal, well-extended and uniformly diversified Pareto optimal front for a given MOP. For the optimization of foothill structure (section 4 ), we have chosen the recently proposed, $\varepsilon$ MOEA (Deb et al., (2003)) because it achieves all the above desired properties at a minimum computation cost. The code of this algorithms is graciously offered by the authors at URL http://www.iitk.ac.in/kangal/soft.htm.

$\varepsilon$-MOEA is based on the principle of $\varepsilon$-dominance, that relaxes the strict Pareto-dominance principle. It uses two co-evolving populations: an EA population and an archive population $\mathrm{A}$, and is a steady-state EA.. The initial archive population $\mathrm{A}$ is assigned from the initial EA population using $\varepsilon$-non-dominated solution.
Thereafter two solutions are selected, one from the EA population using tournament selection and the other from archive A using random selection. From those parents, an offspring $\mathrm{O}$ is created, and is used to update the EA population on the basis of strict dominance, and the archive population on the basis of $\varepsilon$-dominance. Only one solution in each hyper-box on the Paretooptimal front is allowed, to favor diversity.

\section{First Results in Geological Modeling}

\subsection{The Geological Identification Problem}

For identifying a foothill structure, a purely geological inverse problem is defined. In this inverse problem, the unknowns are the parameter of a structure, and the data are layer dip or fault location measured on the topographical surface or along a well (Figure 3). Experimental results are being presented on artificial problems: the "experimental" data are computed on a model that is in itself the result of an evolution using the Contreras kinematic model starting from a known initial configuration. Two models have been considered so far, a 3 fault and a 7 fault models.

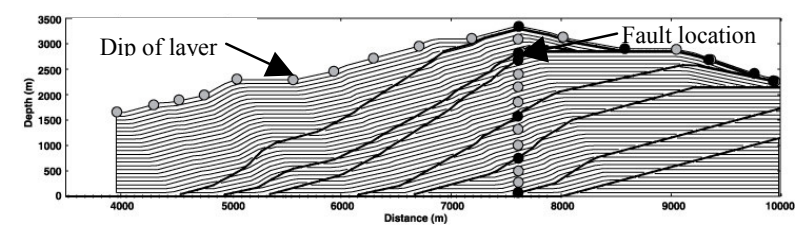

Figure 3 Data are the measured fault location (dark circles) and the dip of the layers (light circle) along surface and well.

\subsection{The Evaluation Functions}

This is the most costly and important step for real applications. Contreras and Suiter (1990), kink model is used for the deformation of the model from the initial state to final state. After deformation, dip and fault location parameters are evaluated for optimization purpose. In general, dip of faults, stratigraphic unit, and fault location are easily obtained from the field observations, well logs (dip-meter) or by remotes sensing data. For the evaluation of a model, a leastsquares criterion measures the discrepancy between the field data and corresponding dip and faults location on 
the current model. The relation is shown by the following equation.

$f 1=\sqrt{\sum_{i=0}^{n d}\left(D_{i p} o b_{i}-D_{i p} g_{-} e n_{i}\right)^{2}}$

$f 2=\sqrt{\sum_{i=0}^{n f}\left(\text { Fault }_{\text {position }} O b_{i}-\text { Fault }_{\text {position }} \text { gen }_{i}\right)^{2}}$

The computational cost of the evaluation function is hence negligible when compared to that of the morphogenesis process (the Contreras model that computes the deformation of the subsurface structure). For instance, for the experiments presented in next subsections, the total computational cost on a Pentium $3.4 \mathrm{GHz}$ is about 4 hours.

\subsection{A Three Fault Model}

A target mountain front model, with five layers and three faults, is shown in figure 4. Because this model was generated from an initial genotype (as described in section 3.1) with the Contreras model, it is the exact solution to the identification problem. On this example, data is only available at the surface, light circles representing dip information, dark circles fault locations.
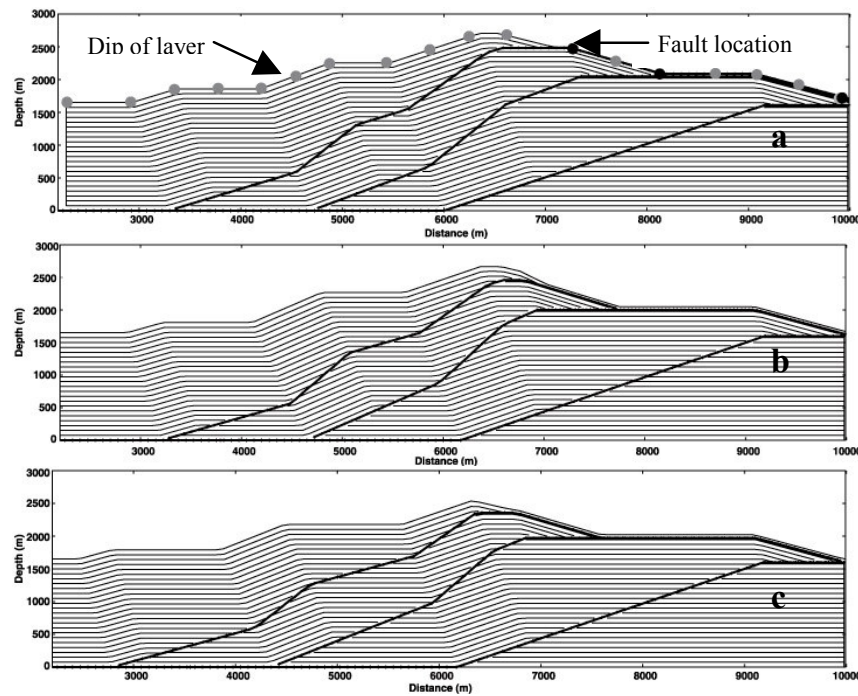

Figure 4 Good results on the 3-faults model: (a) is the target structure. (b) and (c) are result structures. Structure (b) is similar to target structure in all respects. Structure (c) is also similar to the target structure but fault locations are slightly different. However, both structures coincide with the target at the observation points.
For this experiment, the population size was 30 , archive size 30 , and number of generations 300 , and, as mentioned in section 3, the representation was restricted to fixed-length 3 fault genotypes.

Figure 4 shows some identified models that are very similar to the target model. Model 4(b) is similar to the target model in terms of surface and subsurface fault location and topography, while model 4(c) is also similar to the target structure but its fault positions are slightly different.

However, some others models have also be obtained, which are numerically quasi-optimal too, but visually quite different from the target model (see figure 5). This leads to the conclusion that the problem is underdetermined: we need more information in order to uniquely define a solution.
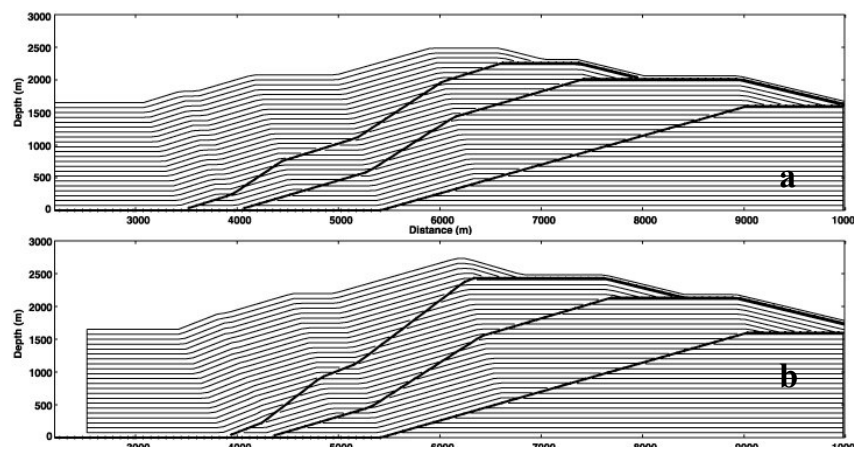

Figure 5 (a) and (b) are the others optimized models. The models (a) and (b) are similar along the topographic surface, but visually different below.

\subsection{Results on the Seven Fault Model}

A more complex seven fault model is shown in figure 6(a). On this model the observation points are located both on the surface and in a well. For this experiment population size was 50 , archive size 50 , and number of iterations was 500. The identified models are shown in figure 6(b) and (c). Again, they are very similar to the target model. In figure 6(b), the fourth fault is visually different from its analogue in the target model but the rest of the faults are both numerically and visually similar. Figure 6(c) also looks very similar to the target but for the sixth and fourth faults. Here also, as can be seen on figure 7 , some identified models are quite 
different from the target model - though being quasioptimal numerically, i.e. the available data on the surface and in the well are almost perfectly fitted.

This situation is very typical of ill-posed problems: we do not have sufficient information to ensure the uniqueness of the solution.

Remember that some basic geometry constraints have been added to the representation (e.g. successive faults first segment length will be more than the previous one, see Section 3.1). Such additional knowledge did prove beneficial: without this information, the algorithm need around 1000 iterations to converge while only around 300 with the constraints. However, higher order of information like seismic, gravity or magnetic data, seem necessary to improve the results.
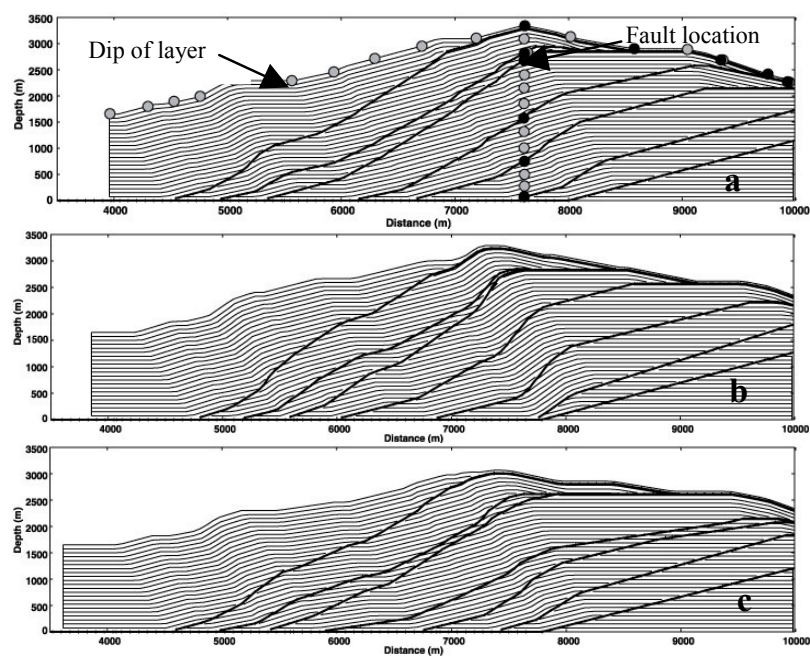

Figure 6 (a) is the target structure, (b) and (c) are the numerically optimised structures. In spite of minor differences, models (b) and (c) are fairly similar.
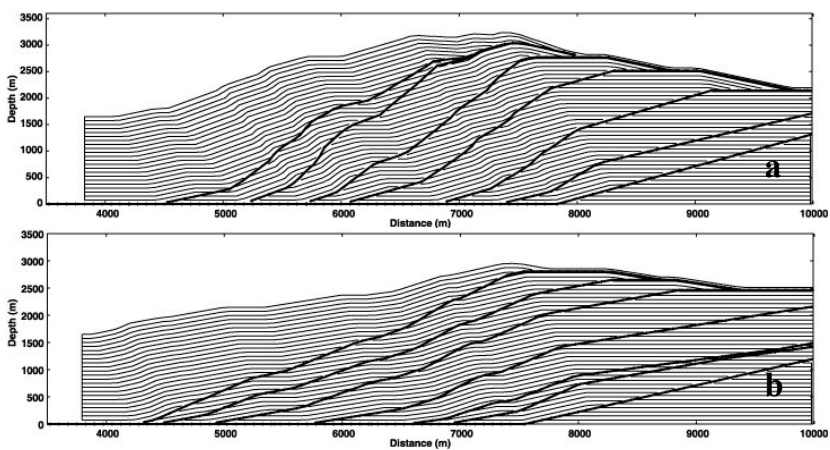

Figure 7 (a) and (b) are other numerically optimal structures for the 7-fault model. But models 7(a) and 7(b) are obviously different from the target mode 6(a). However they fit rather nicely available dip and fault position data as well.

\section{Conclusions and further directions}

The complexity and ambiguity of mountain fronts pose a significant challenge for both geologist and geophysicist in determining the geological and geophysical parameters. In traditional approach mountain front identification needed either rigorous human interaction because of absence of established numerical criteria to act as an inversion target, noisy surface and subsurface data and little prior information, or lead to geologically unrealistic results because, except for very simple regions, the geometry of the subsurface structure cannot be accurately predicted.

We have proposed a new representation for subsurface structure that has both advantages: it is geologically relevant (all structures are balanced), and it does not require human guesses about the geometry of the unknown structure. Moreover, this is the first time, to the best of our knowledge, that multiobjective optimization is applied to a subsurface identification problem. The first results, obtained on synthetic geological identification problems, show the power of the proposed representation, even if we restricted this representation to fixed length for those preliminary experiments.

However, those first results also made it obvious that the geological identification problem is ill-posed, and that more data is needed in order to reach good solutions with more robustness. On-going work is indeed dedicated to adding the seismic data that are available. However, the computation of the geophysical part of the fitness will now rely on some heavy numerical simulation and the overall computational cost will be greater by one order of magnitude. One critical issue will then be how to balance the workload between the purely geological and the geophysical objectives.

Another direction is of course to experiment the full power of the proposed representation with respect to the variable number of faults: at the moment, though we claim that no expert knowledge is needed, we did 
specify the correct number of faults because we used synthetic data. Though there are some good hints about the number or faults thanks to well information, there is always a chance that we miss some faults, or fail to connect the same faults in two different wells - and using the variable-length representation will then make full sense.

\section{References:}

1. Allmendinger, R.W. (1998) "Inverse and forward numerical modeling of trishear fault-propagation folds". Tectonics 17 (1998), 640-656.

2. Beyer Hans-Georg, Deb K (2001) "On self-adaptive features in real-parameter evolutionary algorithms" IEEE Trans. Evolutionary Computation 5(3): 250-270

3. Boyer, S.E., and Elliott, D. (1982) "Thrust systems": American Association of Petroleum Geologists Bulletin, v. 66, 1196-1230

4. Boschetti, F., Dentith, M.C. \& List, R.D. (1996) "Inversion of seismic refraction data using genetic algorithms", Geophysics, 61, 1715-1727.

5. Chester J.S. (2003) "Mechanical stratigraphy and fault-fold interaction, Absaroka thrust sheet, Salt River Range" Wyoming, Journal of Structural Geology 25 (2003), 11711192.

6. Contreras, J., Suiter, M. (1990) "Kinematic modeling of cross-sectional deformation sequences by computer simulation". Journal of Geophysical Research 95, 2191321929.

7. Contreras J. (1991) "Kinematic modeling of cross-sectional deformation sequences by computer simulation: coding and implementation of the algorithm", Computers \& Geosciences, v.17 n.9, 1197-1217.

8. Contreras, J., Suter, M. (1997) "A kinematic model for the formation of duplex systems with a perfectly planar roof thrust". Journal of Structural Geology 19, 269-278.

9. Coello C.C.A. (1999) "A comprehensive survey of evolutionary-based multi-objective optimization techniques" Knowledge and Information Systems An International Journal 1 (3), 269-308.

10. Dahlstrom C.D.A. (1990) "Geometric constraints derived from the law of conservation of volume and applied to evolutionary models for detachment folding" American Association of Petroleum Geologists Bulletin 74 (1990), pp. 336-344.

11. Deb K. and Agrawal R. (1995). "Simulated binary crossover for continuous search space" Complex Systems, 9(2):115-148.

12. Deb K. (2001) "Multi-Objective Optimization using Evolutionary Algorithms" Wiley, Chichester, UK

13. Deb, K, Mohan, M. and Mishra, S. (2003). "A Fast Multiobjective Evolutionary Algorithm for Finding Well-Spread Pareto-Optimal Solutions" KanGAL Report No. 2003002.

14. Endignoux L, Moretti I., and Roure F., 1989, "Forward modeling of the southern Apennines", Tectonics V8, 5, 1095-1104.

15. Erslev E.A. (1991) "Trishear fault-propagation folding" Geology 19 pp. 617-620.

16. Fischer, M.P., Jackson, P.B., (1999) "Stratigraphic controls on deformation patterns in fault-related folds: a detachment fold example from the Sierra Madre Oriental, northeast Mexico" Journal of Structural Geology 21, 613-633.

17. Hardy, S. (1995) "A method for quantifying the kinematics of fault-bend folding"Journal of Structural Geology 17, 1785-1788.
18. Jamison W.R. (1992)" Stress controls of fold thrust style" Thrust Tectonics, Chapman and Hall, London (1992), 155164.

19. Jamison W.R. (1997) "Quantitative evaluation of fractures on Monkshood anticline, a detachment fold in the Foothills of Western Canada" The American Association of Petroleum Geologist Bulletin 81 (1997), 1110-1132.

20. Mansanné, F., and Schoenauer, M., (2002), "An automatic geophysical inversion procedure using a genetic algorithm" Soft computing for reservoir characterization and modeling : Physica-Verlag, Heidelberg, 331-335

21. Mitra, S. (1988) "Three-dimensional geometry and kinematic evolution of the Pine Mountain thrust system, Southern Appalachians" Geological Society of America Bulletin, v. 100, 72-95.

22. Mitra, S. (1992) "Balanced structural interpretations in fold and thrust belts" Structural geology of fold and thrust belts, Johns Hopkins University Press, Baltimore, p. 53-77.

23. Moretti I. and Larrère M., 1989, "LOCACE: ComputerAided Construction of Balanced Geological Crosssections", Geobyte, Oct. 1-24.21.

24. Morgan J.K.and Karig D.E. (1995) "Kinematics and balanced cross-section across the toe of the eastern Nankai accretionary prism" Journal of Structural Geology 17 , 3145 .

25. Ramsay, J.G. (1992) "Some geometric problems of rampflat thrust models" Thrust tectonics, Chapman-Hall, London, 191-200.

26. Rich, J. L. (1934) "Mechanics of low-angle overthrust faulting as illustrated by Cumberland thrust block, Virginia, Kentucky and Tennessee" Bulletin of the American Association of Petroleum Geologists 18, 1584-1 596.

27. Suppe, J. (1983) "Geometry and kinematics of fault-bend folding" American Journal of Science, v. 283, p. 684-721.

28. Suppe, J. and Medwedeff, D.A. (1984) "Fault-propagation folding" Geological Society of America Abstracts with Programs, v. 16, 670.

29. Suppe, J. and Medwedeff, D.A. (1990), "Geometry and kinematics of fault-propagation folding" Eclogae geologicae Helvetiae, v. 83, 409-454.

30. Tanner, P.W.G. (1992) "Morphology and geometry of duplexes formed during flexural-slip folding" Journal of Structural Geology, v. 14, 1173-1192.

31. Sen, M. K. and P. L. Stoffa, Nonlinear one-dimensional seismic waveform inversion using simulated annealing, Geophysics, 56, 1624-1638, 1991.

32. Schoenauer M.and M. Sebag M, (2002), "Using Domain Knowledge in Evolutionary System Identification" Evolutionary Algorithms in engineering and Computer Science John Wiley

33. Storti F. and Salvini F. (1996) "Progressive rollover faultpropagation folding: a possible kinematic mechanism to generate regional-scale recumbent folds in shallow foreland belts" American Association of Petroleum Geologists Bulletin 80, 174-193.

34. Veldhuizen D. and Lamont G. (2000) "Multiobjective evolutionary algorithms: analysing the state-of-the-art" Evolutionary Computation 8 (2), 125-147.

35. Woodward N.B. (1999) "Competitive macroscopic deformation processes", Journal of Structural Geology 21, $1209-1218$

36. Zoetemeijer, R. (1993) "Tectonic Modeling of Foreland Basins: thin skinned thrusting, syntectonic sedimentation and lithospheric flexure" Ph.D. thesis, University of Amsterdam. 